\title{
Peritonitis with Ascites Following Caesarean Delivery: A Case Report
}

\section{Deepali Ghungrud ${ }^{1}$, Ranjana Sharma², Ruchira Ankar ${ }^{2}$}

Section: Healthcare

Sci. Journal Impact

Factor: 6.1 (2018)

ICV: 90.90 (2018)

(c) (7) (8)

Copyright@IJCRR

\author{
'M.Sc. Nursing Student, Department of Medical-Surgical Nursing, Smt.Radhikabai Meghe Memorial College of Nursing, Datta Meghe \\ Institute of Medical Sciences, (Deemed to be University), Sawangi (M) Wardha, Maharashtra, India; ${ }^{2}$ Associate Professor, Departm- \\ net of Medical-Surgical Nursing, Smt.Radhikabai Meghe Memorial College of Nursing, Datta Meghe Institute of Medical Sciences, \\ (Deemed to be University), Sawangi (M) Wardha, Maharashtra, India.
}

\section{ABSTRACT}

Background: Over the last few decades, the caesarean section has increased all over the world. More issues are likely to be observed as caesarean section rate increases. Infection is the leading cause of maternal morbidity and mortality. Postoperative peritonitis and ascites are very rare complications in lower segment caesarean section delivery. The final cause is unknown, but sometimes it causes due to allergic or inflammatory reaction of the peritoneum. If it occurs after caesarean then creates very serious issues with the woman health.

Case Presentation: Here we are mentioning a very rare surgical case of peritonitis with ascites of unknown cause after lower segment caesarean section delivery. In this case, on physical examination and investigation, it was found that, after the caesarean patient has developed severe abdominal pain, fever, nausea, vomiting, abdominal distension, swelling on face and foot. To overcome this sudden issue, an emergency exploratory laparotomy was done. During the surgical exploration of the abdominal cavity maximum amount of clear fluid was seen and that was aspirated by suctioning and peritoneal lavage was given. There was no sign of tissue or organ damage by surgery or no other abnormality was detected during operation. No bacterial growth observed in fluid cultures on the investigation of fluid drained from the abdomen. The working diagnosis was finalized by surgeon i.e. reactive peritonitis with ascites. After expert surgical management and excellent nursing care patient was discharged with full recovery.

Conclusion: In this study, we mainly focus on expert surgical management and excellent nursing care helped in managing the complicated case very nicely. All the patient response was positive for conservative and nursing management and after treatment, the patient was discharged without any postoperative complications and satisfaction with full recovery.

Key Words: Ascites, Caesarean delivery, Complication, Peritonitis, Postoperative

\section{INTRODUCTION}

Over the last few decades, the caesarean section has increased all over the world. More issues are likely to be observed as caesarean section rate increases. Infection is the leading cause of maternal morbidity and mortality. Maternal sepsis was among the causes of maternal mortality in the last confidential investigation on maternal death ${ }^{1}$.

Postoperative peritonitis or inflammation of the peritoneum and fluid accumulation in the peritoneal cavity is one of the very rare complications of caesarean section delivery. It's a life-threatening situation and is a very serious issue concerning caesarean delivery ${ }^{2}$.
The main cause of peritonitis is mostly bacterial infection, foreign bodies in the peritoneum or any chemical agents. Due to these factors, organs or tissues may perforate and sterile peritoneum becomes infected. Sometimes in this situation, women experience localized peritonitis due to infection of the fallopian tube or a ruptured peritoneum ${ }^{3}$.

In these cases, bleeding or iatrogenic injuries, i.e. any tissue or organ damage of the small and large intestine or urinary tract injury should be eliminated on time to improve patient survival and decrease mortality also. Sometimes, the exact cause cannot be found after an extensive diagnostic work-up. In these cases, very rarely idiopathic allergic and inflammatory peritoneal reaction may be the final diagnosis ${ }^{4}$.

\section{Corresponding Author:}

Deepali Ghungrud, M.Sc. Nursing Student, Department of Medical-Surgical Nursing, Smt.Radhikabai Meghe Memorial College of Nursing, Datta Meghe Institute of Medical Sciences, (Deemed to be University), Sawangi (M) Wardha, Maharashtra, India; Email: ghungruddeepali@ gmail.com

ISSN: 2231-2196 (Print)

Received: 15.06 .2020
ISSN: 0975-5241 (Online)

Revised: 06.08 .2020
Accepted: 23.09 .2020
Published: 27.10 .2020 


\section{CASE HISTORY}

A surgical case was taken by Acharya Vinoba Bhave Rural Hospital, Datta Meghe Institute of Medical Sciences, (Deemed to be University), Sawangi (Meghe), Wardha, Maharashtra, India. After caesarean section delivery, this complicated case was taken care of nicely by the hospital because of expert surgical team management and excellent nursing care.

\section{Patient information}

We report a 26-year-old- woman was pregnant with her first child, complete pregnancy period and all routine laboratory blood test was normal. During delivery an emergency lower segment caesarean section delivery was done at full term, the postoperative period was good. Hence, she was discharged on the $7^{\text {th }}$ day after recovery. But after 2 months patient suddenly developed severe abdominal pain. Generalized pain was dull, aching, and also she had a history of multiple episodes of nausea and vomiting. Vomitus had only fluid, blood was not found. She also had diarrhoea, fever $\left(38.5^{\circ} \mathrm{C}\right)$, swelling on face, hand and foot, and abdominal distension. With all these complaints she was admitted in the surgery ward.

Patient belonged to the middle-class family. Her family members had no complaints of communicable and non-communicable diseases. She maintained a good interpersonal relationship with family members and relatives and neighbours also. But due to this condition, she had developed postpartum symptoms like irritability, anxiety, crying and restlessness and found it difficult in bonding with her baby. She was behaving like this due to separation from her 2-month child. Previously and at the time of admission, the patient doesn't have the communicable and non-communicable disease.

After admission, Ryle's tube intubation was inserted. On admission, her vital sign was normal but systolic and diastolic blood pressure was $90 / 60 \mathrm{~mm}$ of $\mathrm{Hg}$, while abdominal on examination was distended. Overall, this condition was managed by administration of intravenous fluid and antibiotic treatment. Blood investigation and the ultrasonography were done, ultrasonography report impression was Hepatosplenomegaly with ascites.

\section{Physical Examination}

Her Physical examination was done and on examination caesarean scar was present on the abdomen. The abdomen was distended and she had swelling on face, hand, and foot. Other than there was no abnormality was detected.

\section{Diagnostic assessment}

The laboratory test was conducted and results were: Hemoglobin $8.9 \mathrm{gm} / \mathrm{dl}(12.1-15.1 \mathrm{gm} / \mathrm{dl})$, and total leukocyte count was 17,000 cell $/ \mathrm{m} 3\left(5000-11,000 \mathrm{cell} / \mathrm{m}^{3}\right)$, urine analysis result was creatinine $2.4 \mathrm{mg} / \mathrm{dl}(0.6-1.4 \mathrm{mg} / \mathrm{dl})$, and blood urea nitrogen level was $63 \mathrm{mg} / \mathrm{dl}(8-25 \mathrm{mg} / \mathrm{dl})$, sodium level $135(135-145 \mathrm{mEq} / \mathrm{l})$ and potassium $4.3 \mathrm{mEq} / 1$ (3.5-4.8 mEq/l) and serum albumin level was $2.3 \mathrm{mg} / \mathrm{dl}$. Serum bilirubin was 1.5 , a liver function test report was shows liver enlarged i.e. $16.2 \mathrm{~cm}$. and radiologist mentioned in ultrasonography report, i.e. Hepatosplenomegaly with ascites.

\section{Preoperative care}

Nasogastric tube intubation was done as per doctors ordered and Ryle's tube aspiration was done 2 hourly. The abdominal girth was recorded 2 hourly. The bladder was catheterized as per doctor's orders and maintained intake and output strictly. Doctors tried to treat this condition with the help of conservative management, i.e. Inj Ceftriaxone $1 \mathrm{gm}$ antibiotic intravenously, Inj. Metronidazole 100ml antimicrobial intravenously, Inj tramadol pain killer in an IV drip, Inj Entomol $100 \mathrm{ml}$ antipyretic intravenously given, but the only operation was next choice for the surgeon to handle this case and before start surgical operation preparation wrote consent was taken by her husband and physical and psychological preparation was done preoperatively. All these conditions were very threatful to the patient because her 2 months the male child was at home and she was so much worried about him and she was continuously asking her husband regarding her child. On observation, she looked very tensed.

But the exact cause was not found and finally, an exploratory laparotomy was done 2400 cubic centimetre straw-coloured accumulated fluids was removed, for investigation and sent that in the laboratory to rule out the cultured. Ovaries, uterus and urinary system organs, i.e. ureter, bladder and gastrointestinal tract were found normal. The abdomen was closed with the use of good quality of suture materials and drain tube.

\section{Postoperative care}

Postoperatively the patient shifted in the surgery Intensive Care Unit; semi fowler's position was given, 4 hourly Ryle's tube aspiration and abdominal girth were measured. Postoperatively continuous cardiac monitoring was done, Inj. Piptaz 4.45 gm higher antibiotic intravenously, Injection. Metronidazole $100 \mathrm{ml}$ antimicrobial intravenously, Inj Pantoprazole antacid $40 \mathrm{mg}$ intravenously, Inj. Emset $4 \mathrm{mg}$ antiemetic intravenously, Inj Tramadol pain killer in an IV drip, Inj Neomol 100ml antipyretic intravenously given as per doctors ordered.

\section{Nursing management}

Postoperatively the patient was under strict observation of on-duty staff. Intravenous fluid administered as per calculation. Observation and reading of the character of the drainage were done postoperatively. Platelets were given, care of the wound and the daily dressing was done. Drainage care 
was taken and intake and output were maintained 2 hourly. Vital signs were recorded strictly. In the cytology report, no malignant cells were found. Overall her response was positive for treatment and patient condition too improved progressively. The drain output became less and was removed after 3 days. Then the patient was shifted in the surgical ward from surgical ICU after recovery.

Excellent nursing care was given and the patient herself reported to nursing staff that. She was very satisfied with nursing care. Complete discharge procedure was explained by nursing staff to the patient and her family members along with medication prescribed at home as advised by the surgeon. The patient was discharged from the ward after 15 days post laparotomy without any complications.

The patient visited regularly at surgery OPD and also visited the hospital at pediatric OPD for her baby for a daily routine checkup, during this period routine checkup was done and she had no any complaints, therefore no furthermore evaluation was found.

\section{DISCUSSION}

This was a very rare surgical case of peritonitis with ascites after caesarean section delivery and fluid accumulation in the peritoneal cavity after an emergency caesarean section delivery without any signs of bleeding, tissue or organ damage of the small intestine and large intestine or ureter, bladder nor peritoneal contamination. No exact cause was found even after performing a thorough postoperative cytological investigation of the peritoneal fluid. Following exploratory laparotomy and drain of the fluid. No, any additional medical treatment was given other than intravenous antibiotics and antimicrobial injections. Pain killer and antiemetic ${ }^{2}$.

We can notice the development of fluid accumulation in the peritoneal cavity due to an idiopathic allergic condition and inflammatory peritoneal reaction. Till now, there is no any exact information in the literature to assume the possibility of a peritoneal allergic reaction or any other inflammatory reaction due to handling of the peritoneum during caesarean section and chemical agents used at the time of surgery, and in the case where vital organ injury. The cause and effects of the disease have not been identified, it is limited to isolated case reports only. Most of the reports are related to this topic are based on patients undergoing gynaecological procedures 5 .

Postoperative peritonitis i.e. inflammation of peritoneum and ascites fluid collection in the peritoneum cavity of unknown cause has been mentioned after laparoscopic surgeries like Appendicectomy, Cholecystectomy, laparotomy for resection of ovarian cyst and salpingectomy $y^{6,7}$.

The same condition of the previous reports has suggested that if chemical agents used at the time of surgery, then there may be chances of an allergic reaction during laparoscopy or laparotomy (antiseptic peritoneal lavage and methylene blue dye) or some other substances used like carbon dioxide, electricity light or heat, diathermy and latex powder ${ }^{8}$.

But in this patient, the surgeon did not use any types of allergic chemical agents or intraperitoneal cauterization. During the caesarean section, the colour of ascites was not in favour of bacterial ascites ${ }^{4}$. It may occur in an allergic reaction. Patient's low immunity power because her haemoglobin was $8.9 \mathrm{gm} / \mathrm{dl}$.

Sometimes, if an aseptic technique is not followed during surgery, it may result in incision site wound sepsis. Without any organ or tissue damage patient may have post-operative peritonitis, it will happen because of a patient's immune system and allergic reaction or patient may or may not be ane$\mathrm{mic}^{4}$.

Ascites is a medical condition in which there is an abnormal accumulation of peritoneal fluid in the peritoneum cavity, caused due to any serious injury to the small or large intestine and urinary tract injuries. But in her case ascites may be occurred due to portal venous pressure and low plasma protein. But no pieces of evidence were found. If peritoneal irritation found present, then leakage of lymphatic fluid in peritoneal cavity ${ }^{2}$.

While performing surgery a surgeon should be very conscious while handling vital organs to prevent further postoperative complications. The gastrointestinal tract was investigated and there was no sign of perforation was found. The patient had no clinical finding in favour of pancreatitis, preoperatively ultrasonography was done liver was enlarged shown Hepatosplenomegaly with ascites.

Gynaecologist has a major responsibility while performing lower segment caesarean section delivery. During surgery use, non-allergic chemical agents, before starting operation nurse has a responsibility to account all instruments that are necessary for surgery, sponges, and gauze pieces also count. Before the closure of the abdomen, the Surgeon should take feedback from the nurse regarding the counting of instruments, sponges and gauze pieces. The surgeon should be very careful and conscious while handling peritoneum and about tissues or organ damaging. Otherwise, it will be a lifethreatening condition for the patient. The serious complication may cause morbidity and mortality.

\section{CONCLUSION}

Postoperative peritonitis and ascites due to unknown cause after a caesarean section delivery is a rare complication. In this case, the patient was completely investigated and monitored is there any possibility of bleeding of an organ or tissue 
damage of the small intestine and large intestine or urinary tract injury during the caesarean section. To manage all the situation emergency exploratory laparotomy is one of the surgical choices as early treatment and investigation; if the patient developed signs of peritonitis no definitive cause for the fluid accumulation can be found. Then many times this condition can be created through idiopathic allergic or inflammatory reaction of the peritoneum. On observation, it shows that after draining the fluid, patients recover well and no further medical intervention was required.

Overall, this condition was very complicated for the patient. But due to the surgeon's expert team and excellent nursing care her condition was improved. In such type of cases, if a patient diagnosed at an early stage we can prevent morbidity and mortality in women. Otherwise, it will be very serious issues for the patient and patient life can be spoiled.

On admission a 26-year-old- woman condition was lifethreatening. It was complicated to handle because of all these condition patients were so fearful and tensed, the patient also developed postpartum symptoms and hence it was difficult to handle but after treatment and surgery slowly her condition was improved. She had multiple doubts regarding the overall condition and her every doubt were cleared. Excellent nursing care was given and she had given positive feedback to nursing staff, she felt better after surgery and she was satisfied. She always talked to every nursing personnel very nicely and given full co-operation to nursing personnel while giving therapeutic care and she was discharged from hospital with full recovery without any post-operative complication and satisfaction, with happy face along with her family members.

\section{ACKNOWLEDGEMENT}

The author thanks Dr.Seema Singh, Professor, and Principal, Smt.Radhikabai Meghe Memorial College of Nursing. Datta Meghe Institute of Medical Sciences, Sawangi (Meghe) Wardha Maharashtra, India. For her timely support and valuable suggestions. Mrs.Vaishali Taksande, Professor, Dean, Dept. of OBGY, DMIMS (DU) for her continuous support and valuable suggestions. The author also thanks Mrs. Maurya, Professor, Dept. of Child Health Nursing. For her timely supports. Also, special thanks to Dr. Geet Mitha, Assistant Professor, General surgery unit, Acharya Vinoba Bhave Rural Hospital, DMIMS(DU), Sawangi (Meghe), Wardha, Maharashtra India.
Authors are also grateful to authors/editors/publishers of all those articles, journals and books, from where the literature for this article has been reviewed and discussed. The authors are grateful to IJCRR editorial board members and IJCRR team of reviewers who have helped to bring quality to this case report.

\section{Ethical approval}

Not applicable

\section{Patient Inform consent}

While preparing a case report and for publication patient's informed consent has been taken.

\section{Conflict of Interest}

The Author declares that there are no conflicts of interest.

\section{Funding}

Not applicable

\section{REFERENCES}

1. Al Qahtani N. Caesarean section scar dehiscence with peritonitis: Does late surgical intervention minimize the risk of hysterectomy. Int J clinical \& case. 2017; 1(5):105-7.

2. Rabiei M, Ghozat R. An unusual case of postoperative ascites after cesarean section delivery. Case Reports in Clinical Practice. 2016; 1(3):68-70.

3. Daley BJ, Katz J, Anand BS. Peritonitis and abdominal sepsis. Medscape. Updated: Feb. 2015; 23.

4. Gado A, Badawi H, Karim A. An unusual case of peritonitis following caesarean delivery. Alexandria journal of medicine. 2018; 54(4):369-71.

5. Halfon JK. Post-Cesarean Section Peritonitis at a Referral Hospital in Rwanda: Factors Associated (Doctoral dissertation, Duke University).

6. Launey Y, Duteurtre B, Larmet R, Nessler N, Tawa A, Mallédant Y, Seguin P. Risk factors for mortality in postoperative peritonitis in critically ill patients. World journal of critical care medicine. 2017 Feb 4; 6(1):48.

7. SN Chugh. Textbook of Medical-Surgical Nursing. First edition: 2013. Avichal publishing Company; 199-201

8. Mabewa A, Seni J, Chaya PL, Mshana SE, Gilyoma JM. Etiology, treatment outcome and prognostic factors among patients with secondary peritonitis at Bugando Medical Centre, Mwanza, Tanzania. World Journal of Emergency Surgery. 2015 Dec 1; 10(1):47. 